\title{
Imatinib-induced Gastrointestinal Vascular Ectasia in a Patient with Advanced GIST: Case Report and Literature Review
}

\author{
MAHMOUD ABU-AMNA ${ }^{1}$, HALIM AWADIE $^{2}$ and GIL BAR-SELA ${ }^{1}$ \\ ${ }^{1}$ Division of Oncology, Rambam-Health Care Campus and Faculty of Medicine, \\ Technion-Israel Institute of Technology, Haifa, Israel; \\ ${ }^{2}$ Department of Gastroenterology, Rambam-Health Care Campus and Faculty of Medicine, \\ Technion-Israel Institute of Technology, Haifa, Israel
}

\begin{abstract}
Background: Imatinib is generally well tolerated in the treatment of advanced gastrointestinal stromal tumors (GIST). Gastrointestinal vascular ectasia (GIVE) and gastric antral vascular ectasia (GAVE), while rare, are significant under-reported complications of imatinib therapy. Case Report: We present one patient with GIVE complicating imatinib therapy with a literature review of this rare side-effect. Results: A 68-year-old woman was diagnosed with advanced GIST, wild-type CKIT. After 3 months of treatment with imatinib, she had partial response. However, she was diagnosed with GAVE and, later, also with GIVE. During her 3-year imatinib treatment, she suffered from severe anemia and required blood transfusions. Conservative treatments were not helpful and the ectatic lesions resolved only with cessation of imatinib. Conclusion: This confirms a causal relationship between GIVE and imatinib. GIVE and GAVE should be considered possible causes of anemia and upper gastrointestinal bleeding in patients receiving imatinib therapy.

Gastrointestinal stromal tumors (GISTs) are the most common primary mesenchymal tumors of the gastrointestinal (GI) tract with an incidence of 3,000-4,000 cases per year in the USA (1). They occur in the stomach (70\%) and small intestine (10-20\%) and, occasionally, in the rectum, esophagus and colon. The tumor's aggressiveness and metastatic potential are dependent on its size and mitotic index, making these the most reliable prognostic factors (2). GISTs express the cell-surface transmembrane receptor KIT
\end{abstract}

Correspondence to: Prof. Gil Bar-Sela, MD, Division of Oncology, Rambam-Health Care Campus, PO Box 9602, Haifa 31096, Israel. Tel: +972 47773128, Fax: +972 47773168, e-mail: g_barsela@ rambam.health.gov.il

Key Words: Gastric antral vascular ectasia, gastrointestinal hemorrhage, gastrointestinal stromal tumors, gastrointestinal vascular ectasia, imatinib. that has tyrosine kinase activity and is the protein product of the KIT proto-oncogene. There are frequent gain-of-function mutations of KIT in GISTs (3). These mutations result in the constitutive activation of KIT signaling, which leads to uncontrolled cell proliferation and resistance to apoptosis. It has been reported that KIT activation occurs in all cases of GIST, regardless of the mutational status of KIT (3).

Imatinib mesylate is a selective inhibitor of certain protein tyrosine kinases: intracellular ABL kinase, chimeric BCRABL fusion oncoprotein of chronic myeloid leukemia, transmembrane receptor KIT and platelet-derived growth factor receptors (4). Imatinib is the primary treatment for patients with advanced, unresectable or metastatic GIST (5). In a pivotal study of imatinib in advanced GIST, treatment was generally well tolerated, although virtually every patient had at least some mild to moderate grade 1 or 2 adverse events, as defined by the Common Terminology Criteria for Adverse Event (CTCAE) that may be related to therapy. The most common adverse events included edema, most frequently periorbital, nausea, diarrhea, myalgia or musculoskeletal pain, fatigue, dermatitis or rash, headache and abdominal pain. Serious adverse events (SAE) (CTCAE-grade 3 or 4) occurred in $21 \%$ of patients, the most serious of which were gastrointestinal or intra-abdominal hemorrhages in patients with large, bulky tumors, which occurred in approximately $5 \%$ of patients; these hemorrhages were probably related to tumor degeneration induced by imatinib (4).

Gastrointestinal vascular ectasia consisted of several distinct congenital syndromes involving abnormal mucosal or submucosal blood vessels in the GI tract. These vessels may cause recurrent bleeding, rarely massive. Diagnosis is by endoscopy and sometimes angiography. Treatment is endoscopic hemostasis; occasionally, angiographic embolisation or surgical resection may be needed (6).

The current report describes a patient with advanced GIST, treated with imatinib for four years, who developed gastric and duodenal vascular ectasia related to imatinib treatment. 


\section{Case Report}

A 68-year-old woman with a medical history of common variable immunodeficiency 14 years previously has been treated since 2010 in our Immunology Unit, with Gammagard (immune globulin infusion) every 2-3 months according to serum antibody levels. In 2006, she was diagnosed with mucosa-associated lymphoid tissue (MALT) lymphoma, low grade, with diffuse lymph nodes and bone marrow involvement. She was then treated with chemotherapy (rituximab with cyclophosphamide, vincristine and prednisone (R-CVP)) and has been in complete remission since and under active surveillance in our Department of Hematology. Positron emission tomography-computed tomography (PET-CT) examination in July 2011 was normal.

As part of her follow-up, she underwent a PET-CT scan in February 2012 that demonstrated many abdominal masses. Ultrasound (US)-guided biopsy of one of the lesions confirmed the diagnosis of GIST (CD117- and discovered on GIST-1 (DOG1)-positive). Bone marrow biopsy was normal. KIT mutation analysis showed wild-type $c$-KIT.

In April 2012, the patient started treatment with imatinib $400 \mathrm{mg} /$ day (Gleevec, Novartis, East Hanover, NJ, USA). In June 2012, she underwent colonoscopy and esophagogastroduodenoscopy (EGD), as part of the diagnostic work-up for primary GIST. On colonoscopy, two small hyperplastic polyps (3-4 mm) were removed. In EGD, vascular ectasia in the stomach and duodenum were seen (Figure 1). The lesions were spontaneously bleeding. She was referred to argon plasma coagulation (APC), which was not done. Her hemoglobin $(\mathrm{Hb})$ level dropped from $11.7 \mathrm{gr} / \mathrm{dl}$ to $10.4 \mathrm{gr} / \mathrm{dl}$. Follow-up by PET-CT, in July 2012, showed very good partial response with near complete reduction in the pathological uptake. Treatment with imatinib was well tolerated except for edema grade 2 and fatigue grade 1 . Blood tests showed a slow decrease in $\mathrm{Hb}$ level to $7.9 \mathrm{gr} / \mathrm{dl}$ in December 2012. Platelets' count and coagulation tests (prothrombin time and activated partial thromboplastin time (APTT)) were normal. Iron profile test (ferritin, transferrin saturation and iron), vitamin B12 and folic acid were normal. Erythropoietin level was low and the patient started subcutaneous (s.c.) darbepoetin alfa (Arnacep ${ }^{\circledR}$ ) $150 \mathrm{mcg}$ every week, with iron supplements, proton-pump inhibitor (PPI) and blood transfusions every 6-8 weeks.

$\mathrm{Hb}$ level was stable, between 10-11 gr/dl, until August 2013, when the patient began to suffer from severe anemia with the need for weekly blood transfusions. Repeated bone marrow biopsy in December 2013 was normal without evidence of lymphoma. EGD in August 2014 showed gastric antral vascular ectasia (GAVE) (Figure 2).

Between February 2014 and January 2015, the patient underwent six EGDs with APC to control bleeding. There was good remission of the GAVE but new angioectasia lesions appeared. She was referred to video capsule endoscopy (VCE) in November 2014 and diagnosed with gastrointestinal vascular ectasia (GIVE) in the gastric body, fundus and duodenum (Figure 3). With the APC, hemoglobin levels were stable, the need for blood transfusions decreased (approximately every 2-3 weeks) and melena was intermittent. She continued with i.v. iron supplements. During all these years, the patient's disease was stable on PET-CT without F-18 fluorodeoxyglucose (FDG) uptake in the abdominal masses.

It was then agreed to withhold imatinib and to repeat EGD after 8 weeks, in order to test the possibility of the drug as inducer of arteriovenous malformation (AVM) creation. The dose of the PPI drug was also elevated. In March 2015, approximately 2 months after stopping imatinib, the patient underwent follow-up EGD, which showed significant improvement in the ectasia of the gastric body, fundus and duodenum.

$\mathrm{Hb}$ level increased from $8.7 \mathrm{gr} / \mathrm{dl}$ to $10.5 \mathrm{gr} / \mathrm{dl}$ without the need for blood transfusions, iron supplements or erythropoietin. Another interesting finding was an increase in the level of serum IgG, from 400 to 621 after imatinib discontinuation, with a longer duration between Gammagard infusions. In August 2015, PET-CT showed stable disease in the abdominal lesions without FDG uptake; however, a new subcutaneous mass in the right femur was seen, suspected as lymphoma. Biopsy revealed marginal zone lymphoma Ki-67 $5 \%$ without distant metastasis according to PET-CT or bone marrow involvement. She received external radiation therapy with a total dose of $30 \mathrm{~Gy}$ in 15 fractions with 4 cycles of rituximab (Mabthera ${ }^{\circledR}$ ).

PET-CT in February 2016 showed no evidence of active lymphoma but marked progression in the GIST with enlargement of the main multi-lobular mass in the abdomen with new FDG uptake (standardized uptake value $(\mathrm{SUV})=4.9)$ in the peripheral zone of the mass. Hb level was $12.7 \mathrm{gr} / \mathrm{dl}$, with no evidence for GAVE on EGD (Figure 4). Treatment with sunitinb $50 \mathrm{mg} /$ day was started.

\section{Discussion}

GAVE was first described in 1953 in a patient with chronic anemia (7). Via gastroscopy, the antrum was described as 'fiery red' with scattered areas of bleeding and clotted blood. Microscopically, chronic inflammation, as well as submucosal edema and dilated veins, were described. Because of the convergence pattern of the longitudinal antral folds on the pylorus, GAVE has the appearance of stripes on a watermelon on EGD, often called 'watermelon stomach' (8). GAVE under imatinib treatment was initially reported during a 1-year, Phase I/IIa, open-label pilot study of imatinib for a non-malignant condition (9). Twenty systemic sclerosis patients with associated interstitial lung disease 


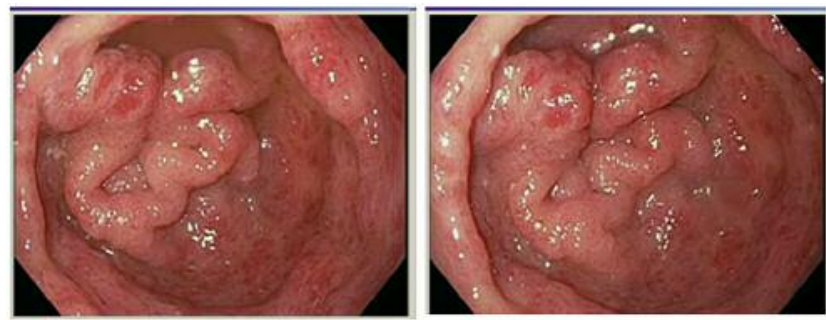

Figure 1. Baseline endoscopic appearance of gastric antral vascular ectasia (GAVE) before argon plasma coagulation (APC) therapy and imatinib cessation.
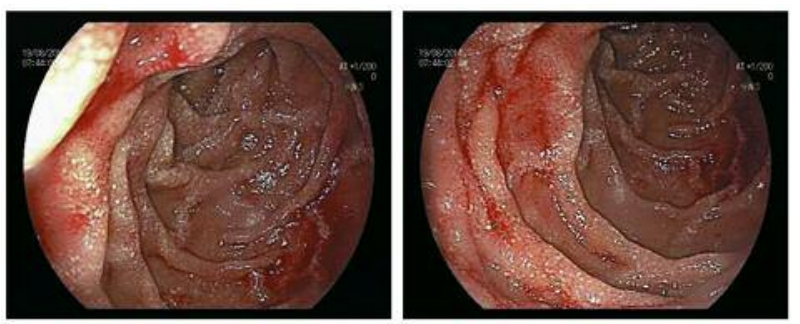

Figure 2. Esophagogastroduodenoscopy in August 2014 during imatinib treatment showed several angiodysplasias with active bleeding in the proximal small bowel found during follow-up gastroscopy for argon plasma coagulation (APC).

were treated with imatinib (up to $600 \mathrm{mg} / \mathrm{day}$ ). Three patients suffered marked anemia, fatigue, muscle weakness and GAVE. In this report, GAVE cannot confidently be attributed to imatinib as the condition may be associated with autoimmune disease.

There are several case reports of GAVE in chronic myeloid leukemia (CML) patients during treatment with imatinib. In the first reported case (10), GAVE did not resolve despite PPI therapy and local cauterization over several years, until imatinib was stopped, suggesting that imatinib was involved in the etiology. Recently, two case series of three CML patients each reported GAVE under treatment with imatinib therapy $(11,12)$. In all cases, GAVE resolved only with cessation of imatinib. In the second series, GAVE resolved when switching from imatinib to nilotinib in one patient and GI bleeding recurred after the readministration of imatinib to another patient (12). All those confirm a causal relationship between GAVE and imatinib.

Imatinib-induced GAVE has been reported in one GIST patient (13). Eight months after imatinib therapy (400 mg/day) was initiated, the patient developed severe anemia, with $\mathrm{Hb}$ $59 \mathrm{~g} / 1$, suggesting acute and severe GI bleeding. EGD revealed GAVE. Imatinib was withheld and the patient was started on PPI treatment. One month later, EGD showed significant
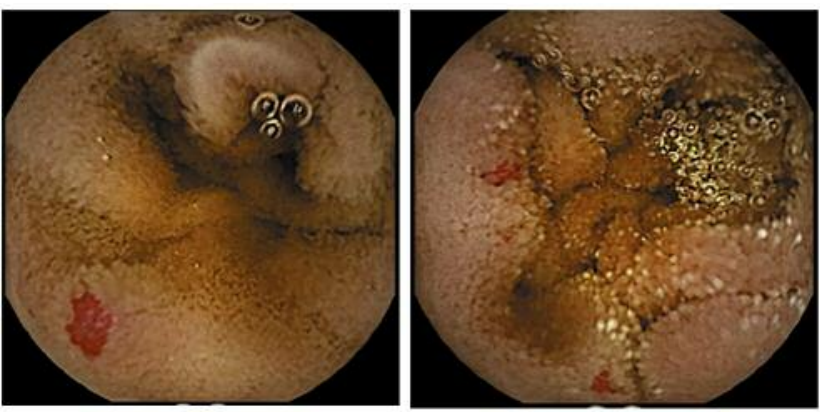

Figure 3. Video capsule endoscopy (VCE) in November 2014 showing multiple diffuse angiodysplasia in the proximal and middle small bowel (white arrows) diagnosed by PillCam SB 3, Given Imaging Ltd.

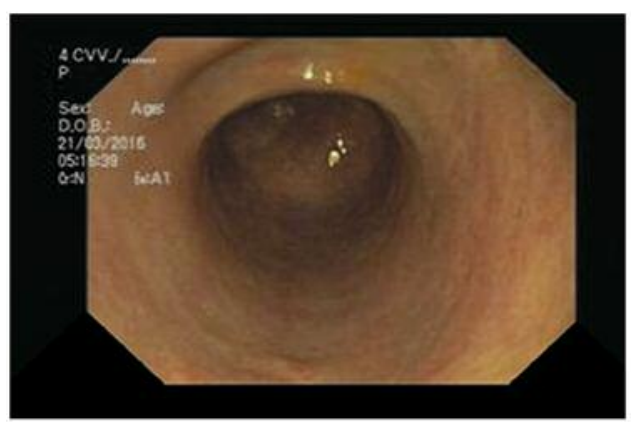

Figure 4. Complete gastric antral vascular ectasia (GAVE) eradication following imatinib cessation.

improvement in the stomach's erythema with resolution of GAVE, indicating that it was likely imatinib-induced, although an alternate unknown etiology that responded to the PPI could not be ruled out. The patient was not re-challenged.

According to Gleevec ${ }^{\circledR}$ prescribing information (14), grade $3 / 4$ hemorrhage was reported in clinical studies in patients with newly diagnosed CML and with GIST. GI tumor sites may be the source of GI bleeds in GIST. In addition, GAVE has been reported in post-marketing experience.

Several treatment strategies for GAVE exist. Symptomatic therapy with multiple blood transfusions and fluid resuscitation aims to correct anemia and maintain hemodynamic stability, respectively. Other medical therapies that were used with variable success rates include corticosteroids (15), tranexamic acid (16) and thalidomide (17). Endoscopic therapy remains the treatment of choice for GAVE. Photocoagulation and argon plasma coagulation have been successful in treating GAVE and abolishing or reducing transfusion requirements (18). For intractable cases, surgical antrectomy is the treatment of last resort. While curative, antrectomy is associated with high morbidity and mortality (8). 
In the current case, GAVE and GIVE did not resolve despite PPI therapy and local endoscopic treatment over several years, until imatinib was stopped, suggesting that this complication most probably took place as a severe adverse reaction to imatinib. To our knowledge, this is the first report in the literature of imatinib-induced gastrointestinal vascular ectasia other than the gastric antrum.

In the current patient, her illness progressed after one year without any treatment for the advanced GIST. Although she had very good response to imatinib treatment, we preferred not to re-challenge with imatinib at the recommended dose (400 mg every day) or a lower dose. The only reported case of imatinib re-challenge caused recurrent GI bleeding (12).

\section{Conclusion}

Vascular ectasia of the gastrointestinal tract is a rare, severe adverse event of treatment with imatinib, regardless of the primary illness or malignancy. Physicians should be aware of this complication that can cause gastrointestinal bleeding, which may be acute or chronic.

\section{Conflicts of Interest}

The Authors have no conflict of interest to declare.

\section{References}

1 Nilsson B, Bümming P, Meis-Kindblom JM, Odén A, Dortok A, Gustavsson B, Sablinska K and Kindblom LG: Gastrointestinal stromal tumors: the incidence, prevalence, clinical course, and prognostication in the preimatinib mesylate era - A populationbased study in Western Sweden. Cancer 103: 821-829, 2005.

2 Samelis GF, Ekmektzoglou KA and Zografos GC: Gastrointestinal stromal tumors: clinical overview, surgery and recent advances in imatinib mesylate therapy. Eur J Surg Oncol 33: 942-950, 2007.

3 Rubin BP, Singer S, Tsao C, Duensing A, Lux ML, Ruiz R, Hibbard MK, Chen CJ, Xiao S, Tuveson DA, Demetri GD, Fletcher $\mathrm{CD}$ and Fletcher JA: KIT activation is a ubiquitous feature of gastrointestinal stromal tumors. Cancer Res 61: 81188121, 2001.

4 Demetri GD, von Mehren M, Blanke CD, Van den Abbeele AD, Eisenberg B, Roberts PJ, Heinrich MC, Tuveson DA, Singer S, Janicek M, Fletcher JA, Silverman SG, Silberman SL, Capdeville R, Kiese B, Peng B, Dimitrijevic S, Druker BJ, Corless C, Fletcher CD and Joensuu H: Efficacy and safety of imatinib mesylate in advanced gastrointestinal stromal tumors. N Engl J Med 347: 472-480, 2002.

5 Soft tissue sarcoma. NCCN guidelines version 2.2016.
6 Sami SS, Al-Araji SA and Ragunath K: Review article: Gastrointestinal angiodysplasia - pathogenesis, diagnosis and management. Aliment Pharmacol Ther 39: 15-34, 2014. doi: 10.1111/apt.12527.

7 Rider JA, Klotz AP and Kirsner JB: Gastritis with veno-capillary ectasia as a source of massive gastric hemorrhage. Gastroenterology 24: 118-123, 1953.

8 Selinger $\mathrm{CP}$ and Ang YS: Gastric antral vascular ectasia (GAVE): An update on clinical presentation, pathophysiology and treatment. Digestion 77: 131-137, 2008. doi: 10.1159/ 000124339 .

9 Khanna D, Saggar R, Mayes MD, Abtin F, Clements PJ, Maranian P, Assassi S, Saggar R, Singh RR and Furst DE: A one-year, phase I/IIa, open-label pilot trial of imatinib mesylate in the treatment of systemic sclerosis-associated active interstitial lung disease. Arthritis Rheum 63: 3540-3546, 2011. doi: 10.1002/art.30548.

10 Alshehry NF, Kortan P and Lipton JH: Imatinib-induced gastric antral vascular ectasia in a patient with chronic myeloid leukemia. Clin Case Rep 2: 77-78, 2014. doi: 10.1002/ccr3.49.

11 Ong J, Yeung D, Filshie R, Hughes TP and Nandurkar H: Imatinib-induced gastric antral vascular ectasia in three patients with chronic myeloid leukaemia. Int J Hematol 102: 639-642, 2015. doi: 10.1007/s12185-015-1824-y.

12 Narukawa K, Kakihana K, Fujiwara T, Kobayashi T, Doki N, Sakamaki $\mathrm{H}$ and Ohashi K: Gastric antral vascular ectasia during the treatment of chronic myelogenous leukemia with imatinib mesylate. Intern Med 55: 69-72, 2016. doi: 10.2169/ internalmedicine.55.4166.

13 Saad Aldin E, Mourad F and Tfayli A: Gastric antral vascular ectasia in a patient with GIST after treatment with imatinib. Jpn J Clin Oncol 42: 447-450, 2012. doi: 10.1093/jjco/hys032.

14 GLEEVEC prescribing information: https://www.pharma.us. novartis.com/product/pi/pdf/gleevec_tabs.pdf

15 Bhowmick BK: Watermelon stomach treated with oral corticosteroid. J R Soc Med 86: 52, 1993.

16 McCormick PA, Ooi $\mathrm{H}$ and Crosbie O: Tranexamic acid for severe bleeding gastric antral vascular ectasia in cirrhosis. Gut 42: 750-752, 1998.

17 Dunne KA, Hill J and Dillon JF: Treatment of chronic transfusion-dependent gastric antral vascular ectasia (watermelon stomach) with thalidomide. Eur J Gastroenterol Hepatol 18: 455456, 2006.

18 Kwan V, Bourke MJ, Williams SJ, Gillespie PE, Murray MA, Kaffes AJ, Henriquez MS and Chan RO: Argon plasma coagulation in the management of symptomatic gastrointestinal vascular lesions: Experience in 100 consecutive patients with long-term follow-up. Am J Gastroenterol 101: 58-63, 2006.

Received July 10, 2016

Revised July 20, 2016

Accepted July 21, 2016 